\title{
How Inflation Is Used To Resolve the Flatness Problem
}

September 9, 2005

Joel Smoller ${ }^{1}$ Blake Temple ${ }^{2}$

To Tai-Ping Liu on the occasion of his sixtieth birthday.

\begin{abstract}
We give a mathematically rigorous exposition of the flatness problem, and show how Guth's original model of inflation is used to resolve it.
\end{abstract}

\section{Introduction}

Inflation is an epoch of explosive increase in scale incorporated into the standard model of cosmology. To quote Guth, the founder of the theory of inflation, (Blau and Guth, Inflationary Cosmology, page 525): "The inflationary universe is a modification of the standard hot big bang model motivated by several flaws that emerge when the standard model is extrapolated backward to very early times. The inflationary model agrees precisely with the standard model description of the observed universe for all times later than about $10^{-30} s$ [after the Big Bang], and all the successes of the standard model are preserved." The four main flaws in the standard model of cosmology that Guth is referring to are the flatness problem; the horizon problem; the density fluctuation problem; and the monopole problem. The interest in inflation rests on the fact that an explosive increase in the scale factor $R(t)$ of the standard FRW model at a very early time gives a plausible resolution of these four problems, [1].

The idea that the universe went through a period of rapid expansion early on is perhaps more fundamental than any of the physical theories that have been devised to explain such an expansion. Most compelling is the fact

\footnotetext{
${ }^{1}$ Department of Mathematics, University of Michigan, Ann Arbor, MI 48109; Supported in part by NSF Applied Mathematics Grant Number DMS-010-3998.

${ }^{2}$ Department of Mathematics, University of California, Davis, Davis CA 95616; Supported in part by NSF Applied Mathematics Grant Number DMS-040-6096.
} 
that the microwave background radiation coming from different directions is at the same temperature even though a calculation using the standard model of cosmology without inflation shows that radiation from opposite directions was some seventy or more horizon distances apart at the time the radiation was emitted, (that is, separated by some seventy times light travel distance since the Big Bang), [1] - this is the horizon problem. Thus, assuming thermal contact at an early time, it is easy to believe that radiation coming in from different directions must have been pulled apart by some sort of expansion at some early time. In Guth's original model of inflation, an argument based on invariance principles leads to the conclusion that the stress tensor during the inflationary epoch is of the form of a cosmological constant; that is, $T_{i j}=\lambda g_{i j}$, where $\lambda$ is constant, and $\rho_{*}=-\lambda>0$ is the energy density, c.f. [1], page 541. Such a source term is then shown to produce an exponential increase in the cosmological scale factor thus providing the "inflation".

In this paper we give a mathematically precise discussion of the flatness problem. A mathematically rigorous formulation of the flatness problem in the standard model of cosmology is given in Section 2. In Section 3 we derive exact formulas for the inflationary FRW metrics, (that is, FRW metrics when the source term is $T_{i j}=-\rho_{*} g_{i j}$ ), and based on these formulas we show rigorously how inflation is used to resolve the flatness problem. This paper is preliminary to a future publication in which we will investigate the connection between the inflationary spacetimes and the finite mass shock wave cosmology introduced in [5].

\section{The FRW Metric}

The standard model of cosmology is based on the assumption that the universe is globally homogeneous and isotropic about every point. This implies that in co-moving coordinates, the gravitational metric tensor takes the form of an FRW metric, [7],

$$
d s^{2}=-d t^{2}+R(t)^{2}\left\{\frac{d r^{2}}{1-k r^{2}}+r^{2} d \Omega^{2}\right\} .
$$

Here $R(t)$ is the cosmological scale factor, $k$ is the curvature parameter, $\frac{k}{R^{2}}$ is the spatial curvature at each fixed time $t$, and $d \Omega^{2}$ is the standard metric on the unit 2-sphere. Note that the metric is invariant under the scaling

$$
R \rightarrow \omega R
$$




$$
\begin{aligned}
& r \rightarrow \omega^{-1} r \\
& k \rightarrow \omega^{2} k,
\end{aligned}
$$

for any positive constant $\omega$. Under this scaling both $\bar{r}=r R$ and $k / R^{2}$, (the scalar curvature of the 3 -surface $t=$ const.), are invariant, and we can rescale $k$ to one of the values $\{-1,0,1\}$, or we can set $R\left(t_{0}\right)=1$ at any given time $t_{0}$, (but not both unless $k=0$ ).

Assuming the stress tensor $T$ takes the form of a perfect fluid, we have

$$
T_{i j}=\left(\rho c^{2}+p\right) u_{i} u_{j}+p g_{i j}
$$

where $\rho c^{2}$ is the energy density, $p$ is the pressure, $u^{i}$ is the $i^{\prime} t h$ component of the 4-velocity of the fluid, $g_{i j}$ is the gravitational metric tensor, and we use the convention that we take the speed of light $c=1$ and Newton's constant $\mathcal{G}=1$ when convenient. Putting (2.1) and (2.3) into the Einstein equations

$$
G=\kappa T, \quad \kappa=\frac{8 \pi \mathcal{G}}{c^{4}},
$$

and assuming the fluid is co-moving with the FRW metric, we obtain the FRW equations for $R$ and $\rho$ :

$$
\begin{aligned}
H^{2} & =\frac{\kappa}{3} \rho c^{2}-\frac{k}{R^{2}} \\
\dot{\rho} & =-3(\rho+p) H,
\end{aligned}
$$

where $H \equiv H(t)$ denotes the Hubble "constant",

$$
H=\frac{\dot{R}}{R}
$$

and overdot denotes differentiation with respect to FRW time $d / d(c t)$. Equations (2.5), (2.6) close when an equation of state $p=p(\rho)$ is specified. When we take $p=-\rho$, the stress tensor (2.3) reduces to

$$
T_{i j}=-\rho g_{i j}
$$

and since (2.6) then implies that $\rho=\rho_{*}=$ const., it follows that the case of a cosmological constant $\lambda=-\rho_{*}$ is equivalent to setting $p=-\rho$ in (2.3). In any case, if $p=p(\rho)$ is specified, then a measurment of the density $\rho\left(t_{0}\right)$ and 
Hubble constant $H\left(t_{0}\right)$ at an initial time $t=t_{0}$, together with an arbitrary assignment of $R\left(t_{0}\right)=R_{0}$ determines $k$ through (2.5), and then system (2.5), (2.6) determines a unique evolution for $t \geq t_{0}$. Note that the curvature $\frac{k}{R^{2}\left(t_{0}\right)}$ is determined by the density and Hubble constant through (2.5), and these three quantities are invariant under the scaling (2.2)).

We need the following result concerning general solutions of $(2.5),(2.6)$ :

Lemma 1 Let $\rho(t), p(t)$ and $R(t)$ denote a positive solution of (2.5), (2.6) defined on some interval of positive times $I=(a, b)$. Assume that $\dot{R}>0$ for all $t \in I$, and assume there exists a constant $\sigma \geq 0$ such that the pressure $p$ and density $\rho$ satisfy the bound

$$
0 \leq \frac{p(t)}{\rho(t)} \leq \sigma
$$

for all $t \in I$. Then for every pair of times $t, t_{0} \in I$ the following inequality holds:

$$
\left(\frac{R_{0}}{R}\right)^{3(1+\sigma)} \leq \frac{\rho}{\rho_{0}} \leq\left(\frac{R_{0}}{R}\right)^{3}
$$

Proof: The proof follows easily from (2.6).

The flatness problem, (which Guth attributes to Dicke and Peebles, [3]), concerns the density parameter $\Omega(t)$ defined to be the ratio of the density $\rho(t)$ to the critical density $\rho_{c}(t)$,

$$
\Omega(t)=\frac{\rho(t)}{\rho_{c}(t)}
$$

where $\rho_{c}(t)$ is the density one would find in the universe at a given value $H(t)$ of the Hubble constant, assuming $k=0$ at that time. By $(2.5), \rho_{c}(t)=$ $\frac{3}{\kappa} H(t)^{2}$, and,

$$
\Omega(t)=\frac{\frac{\kappa}{3} \rho}{H^{2}}=1+\frac{k}{R^{2} H^{2}} .
$$

The flatness problem is the problem of why the universe has a value of $\Omega(t)$ so close to unity at present time, (to within about one percent according to recent observations). The problem is that $\Omega(t)=1$ can be viewed as an unstable equilibrium point in the standard model. The following theorem can be used to give a mathematically precise formulation of the flatness problem in the standard model: 
Theorem 1 Let $\rho(t), H(t)$ and $R(t)$ be any solution of (2.5), (2.6) defined in an interval of positive time $t \in I \equiv(a, b)$. Then for any two points $t, t_{0} \in I$, the following identity holds:

$$
\Omega(t)-1=\frac{\Omega\left(t_{0}\right)-1}{\Omega\left(t_{0}\right)\left[\left(\frac{R}{R_{0}}\right)^{2}\left(\frac{\rho}{\rho_{0}}\right)-1\right]+1} .
$$

Proof of Theorem 1: Start with (2.5) to get

$$
H^{2}=\frac{\kappa}{3} \rho_{0}\left(\frac{\rho}{\rho_{0}}\right)-\frac{k}{R^{2}}
$$

Using (2.5) in the form $\rho_{0}=\frac{3}{\kappa}\left[1+\frac{k}{H_{0}^{2} R_{0}^{2}}\right] H_{0}^{2}>0$ to eliminate $\rho_{0}$ from (2.14), we can solve for $\Theta$ to get,

$$
\begin{aligned}
\Theta & =\left[1+\frac{k}{H_{0}^{2} R_{0}^{2}}\right]\left(\frac{R_{0}}{R}\right)^{2}\left(\frac{\rho_{0}}{\rho}\right)-\frac{k}{H_{0}^{2} R_{0}^{2}} \\
& =\Omega\left(t_{0}\right)\left[\left(\frac{R_{0}}{R}\right)^{2}\left(\frac{\rho_{0}}{\rho}\right)-1\right]+1,
\end{aligned}
$$

where we have used (2.12) for $\Omega\left(t_{0}\right)-1=\frac{k}{H_{0}^{2} R_{0}^{2}}$. Using (2.15) in (2.16) gives (2.13).

We can use (2.13) to see the sense in which $\Omega-1=0$ can viewed as an unstable equilibrium point for (2.5), (2.6). Namely, write (2.13) in the form

$$
\Omega\left(t_{0}\right)-1=(\Omega(t)-1) \Theta
$$

Now assuming that $0 \leq \frac{p}{\rho} \leq \sigma$ for some postive constant $\sigma$, (a reasonable assumption in the standard model), we can apply (2.10) to (2.13) to obtain the inequality

$$
\Theta=\Omega\left(t_{0}\right)\left[\left(\frac{R}{R_{0}}\right)^{2}\left(\frac{\rho}{\rho_{0}}\right)-1\right]+1 \geq \Omega\left(t_{0}\right)\left[\left(\frac{R_{0}}{R}\right)^{1+3 \sigma}-1\right]+1
$$

From (2.16), (2.17) we conclude that $\Theta \rightarrow+\infty$ as $R \rightarrow 0$. Thus (2.16), (2.17) gives an exact sense in which $\Omega=1$ is an unstable equilibrium in the standard model. In particular, we can state the flatness problem as follows: The density parameter $\Omega(t)$ measures the ratio of the density to the 
expansion rate at a given time in an FRW solution. Now one would expect that the density and expansion rate at that density would be independent unless there were a mechanism to correlate them. Barring such a mechanism, one would expect to find a large range of equally likely values of $\Omega(t)$ at an early time $t$ when $\Theta$ could be arbitrarily large. Thus (2.16), which implies that $\Omega(t)-1$ is $\Theta$ times closer to zero than $\Omega\left(t_{0}\right)-1$, thereby also implies that it would be highly unlikely to find the value $\Omega\left(t_{0}\right)$ as close to unity as we find it at present time $t=t_{0}$ if a large range of values of $\Omega(t)$ were equally likely at time $t$. Assuming that $R \rightarrow 0$ as $t \rightarrow 0$, this becomes more unlikely as we take $t$ smaller and smaller because the amplification factor $\Theta$ tends to infinity as $t$ tends to zero.

\section{The Inflationary FRW Metric}

In this section we give exact solutions of the FRW equations (2.5), (2.6) for arbitrary values of the curvature parameter $k \in \mathbf{R}$ in the case of inflation, the case when $T_{i j}=-\rho_{*} g_{i j}$ for some $\rho_{*}=$ const., or equivalently, the case of a perfect fluid with $p=-\rho$. We then discuss the flatness problem, and give rigorous estimates that show how Guth's original inflationary epoch is used to resolve it. Our discussion is based on the following theorem:

Theorem 2 The general solution of the FRW equations (2.5), (2.6) when $T_{i j}=-\rho g_{i j}, \rho_{*}>0$, is given by,

$$
\begin{gathered}
\rho \equiv \rho_{*}=\text { const. }>0 \\
R(t)=\frac{k}{4 \gamma^{2} C} e^{-\gamma c t}+C e^{\gamma c t},
\end{gathered}
$$

where

$$
\gamma=\sqrt{\frac{\kappa \rho_{*} c^{2}}{3}}
$$

and $\rho_{*}>0$ and $C$ are constants of integration.

Proof: Direct substitution of (3.1), (3.2) into (2.5) and (2.6) verifies the theorem.

Note that the placement of the arbitrary constant of integration $C$ in (3.2) nicely displays the continous dependence of solutions in limit $k \rightarrow 0$. 
Note also that when $k>0,(3.2)$ implies that $R(t)>0$ if and only if $C>0$, and in this case $R(t)$ takes a minimum value at

$$
t_{*}=\frac{1}{2 c \gamma} \ln \frac{|k|}{4 \gamma^{2} C^{2}} .
$$

On the other hand, when $k<0,(3.2)$ implies that $\dot{R}>0$ if and only if $C>0$, and in this case $R(t)>0$ if and only if $t>t_{*}$. Thus we have:

Proposition 1 Assume $C>0$. Then solutions of (3.2) satisfy $t>t_{*}$ if and only if both $R(t)>0$ and $H(t)>0$.

Note that $C$ scales like $C \rightarrow \omega^{-1} C$ under the scaling (2.2).

Assume now that $C>0$ and let $t=t_{-}>t_{*}$ denote the starting time of inflation. We can non-dimensionalize (3.2) with respect to the scaling (2.2) by dividing through by the value $R\left(t_{-}\right) \equiv R_{-}$and write

$$
\hat{R}(t)=\frac{\hat{k}}{4 \gamma^{2} \hat{C}} e^{-\gamma c t}+\hat{C} e^{\gamma c t}
$$

where

$$
\begin{aligned}
\hat{R} & =\frac{R}{R_{-}}, \\
\hat{k} & =\frac{k}{R_{-}^{2}}, \\
\hat{C} & =\frac{C}{R_{-}} .
\end{aligned}
$$

The constant $\hat{C}$ in (3.5) can be evaluated in terms of the Hubble constant $H_{-}$and curvature $\hat{k}$. More useful is the next result in which $\hat{C}$ is eliminated from (3.5) in favor of the density parameter $\Omega$ at $t=t_{-}$.

Theorem 3 Let $R(t)$ be the solution of the FRW equations (2.5), (2.6) when $T_{i j}=-\rho_{*} g_{i j}, t \in \mathbf{R}$, such that at time $t=t_{-}$, we have $\rho\left(t_{-}\right)=\rho_{*}, \Omega\left(t_{-}\right)=$ $\Omega_{-}$, and we set $R\left(t_{-}\right)=R_{-}>0$. Then

$$
\hat{R}(t)=\left\{\frac{\sqrt{\Omega_{-}}-1}{2 \sqrt{\Omega_{-}}}\right\} e^{-\gamma c\left(t-t_{-}\right)}+\left\{\frac{\sqrt{\Omega_{-}}+1}{2 \sqrt{\Omega_{-}}}\right\} e^{\gamma c\left(t-t_{-}\right)} .
$$


Moreover, this is equivalent to

$$
\Omega(t)-1=\frac{4}{\frac{\sqrt{\Omega_{-}}-1}{\sqrt{\Omega_{-}}+1} e^{-2 \gamma c\left(t-t_{-}\right)}+\frac{\sqrt{\Omega_{-}}+1}{\sqrt{\Omega_{-}}-1} e^{2 \gamma c\left(t-t_{-}\right)}-2} .
$$

Proof: Let

$$
a=\frac{\hat{k}}{\gamma^{2}}
$$

so that we can rewrite $(3.5)$ as

$$
\hat{R}(t)=\frac{a}{4} e^{-\gamma c(t-\tau)}+e^{\gamma c(t-\tau)},
$$

where we set $\hat{C}=e^{-\gamma c \tau}$ because $\hat{C}>0$. Thus

$$
\begin{aligned}
H_{-} \equiv H\left(t_{-}\right) & =\frac{\hat{R}\left(t_{-}\right)}{\dot{\hat{R}}\left(t_{-}\right)}=\gamma \frac{-\frac{a}{4}+b^{2}}{\frac{a}{4}+b^{2}}, \\
b & =e^{\gamma c\left(t_{-}-\tau\right)} .
\end{aligned}
$$

Now solving (3.12) for $b^{2}$ and using (2.12) we obtain

$$
b^{2}=\frac{a}{4} \frac{\sqrt{\Omega_{-}}+1}{\sqrt{\Omega}-1} .
$$

Putting (3.14) into (3.11) we obtain

$$
\hat{R}(t)=\operatorname{sign}(a) \sqrt{\frac{a}{4} \frac{\sqrt{\Omega_{-}}-1}{\sqrt{\Omega_{-}}+1}} e^{-\gamma c\left(t-t_{-}\right)}+\sqrt{\frac{a}{4} \frac{\sqrt{\Omega_{-}}+1}{\sqrt{\Omega_{-}}-1}} e^{\gamma c\left(t-t_{-}\right)},
$$

where we use that $\operatorname{sign}(a)=\operatorname{sign}\left({\sqrt{\Omega_{-}}}_{-}-1\right)$. In fact, by $(2.5), a=\frac{\Omega_{-}-1}{\Omega_{-}}$, and putting this into (3.15) gives the result (3.9).

To verify (3.10), use (2.5) to obtain,

$$
\begin{aligned}
\Omega(t)-1 & =\left(\Omega\left(t_{-}\right)-1\right) \frac{H_{-}^{2} R_{-}^{2}}{H^{2} R^{2}}=\frac{k}{H^{2} R^{2}}=\frac{\hat{k}}{\gamma^{2} \hat{R}^{2}-\hat{k}} \\
& =\frac{1}{\frac{1}{a} \hat{R}^{2}-1}=\frac{1}{\frac{\Omega_{-}}{\Omega_{-}} \hat{R}^{2}-1},
\end{aligned}
$$


where we have used (3.6)-(3.8), (2.12) and (2.5) in the form $H^{2}=\gamma^{2}-\frac{\hat{k}}{\hat{R}^{2}}$. Putting (3.9) in (3.16) and simplifying gives the final equality (3.10).

Equation (3.10) shows directly how inflation is used to solve the flatness problem. Indeed, an immediate consequence of (3.9) is the following result:

Corollary 1 The inflationary solution $\hat{R}(t)$ given in (3.10) satisfies

$$
|\Omega(t)-1| \leq \frac{8}{e^{2 \gamma c\left(t-t_{-}\right)}}
$$

so long as

$$
t-t_{-}>\frac{\ln (4)}{2 \gamma c}
$$

Proof: Observe that in (3.10), $\left|\frac{\sqrt{\Omega_{-}}+1}{\sqrt{\Omega}_{-}-1}\right| \geq 1$, and the positive and negative exponential terms in the denominator of (3.10) have the same sign. From this it follows that if $e^{2 \gamma c \Delta t} \geq 2$, then

$$
|\Omega(t)-1| \leq \frac{4}{e^{2 \gamma c\left(t-t_{-}\right)}-2} .
$$

Thus if $e^{2 \gamma c \Delta t} \geq 4$, which is (3.18), then $2 \leq \frac{1}{2} e^{2 \gamma c \Delta t}$, and using this in (3.19) one can easily deduce (3.17)

The final theorem in this section gives a estimate for $\Omega\left(t_{0}\right)-1$ at present time $t_{0}$ in terms of the present density $\rho_{0}$, the density at inflation $\rho_{*}$, and the time of inflation $\Delta t$, assuming that after inflation the equation of state satisfies $\frac{p}{\rho} \leq \sigma$ for some positive constant $\sigma$. Interestingly, the estimate shows that the density parameter $\Omega\left(t_{0}\right)-1$ decays to zero at an exponential rate that depends only on the density of inflation and the time of inflation, but is independent of the magnitude of the curvature parameter $k$ which can be arbitrarily large at the start of inflation.

Theorem 4 Assume that an inflationary epoch evolves according to (3.5) between times $t_{-} \leq t \leq t_{+}=t_{-}+\Delta t$ starting at $t_{-}>0$ from initial density and Hubble constant $\rho\left(t_{-}\right)=\rho_{*}>0, H\left(t_{-}\right)=H_{-}>0$, and assume that $R\left(t_{-}\right)=R_{-}>0$ is specified. Let $\rho\left(t_{+}\right)=\rho_{*}, H\left(t_{+}\right)=H_{+}$and $R\left(t_{+}\right)=R_{+}$ be the values then determined by the solution (3.5) at time $t=t_{+}$, and assume that for times $t_{+} \leq t \leq t_{0}$, the solution $R(t)$ continues to evolve as an $F R W$ solution with different equation of state $p=p(\rho)$ starting at $t=t_{+}$with 
initial values $\rho\left(t_{+}\right)=\rho_{+}, H\left(t_{+}\right)=H_{+}$and $R\left(t_{+}\right)=R_{+}$, and ending at time $t=t_{0}$ with values $\rho\left(t_{0}\right)=\rho_{0}, H\left(t_{0}\right)=H_{0}$ and $R\left(t_{0}\right)=R_{0}$. Assume that there exists a positive constant $\sigma$ such that

$$
0 \leq \frac{p(t)}{\rho(t)} \leq \sigma
$$

for all $t_{+} \leq t \leq t_{0}$, and finally, assume that the inflationary epoch is long enough so that

$$
\Delta t>\sqrt{\frac{3}{4 \kappa \rho_{*} c^{2}}} \ln \left\{16\left(\frac{\rho_{*}}{\rho_{0}}\right)^{\frac{1+3 \sigma}{3(1+\sigma)}}\right\} .
$$

Then the following inequality holds:

$$
\left|\Omega\left(t_{0}\right)-1\right| \leq 16\left(\frac{\rho_{*}}{\rho_{0}}\right)^{\frac{1+3 \sigma}{3(1+\sigma)}} e^{-\sqrt{\frac{4 \kappa \rho_{*} c^{2}}{3}} \Delta t} .
$$

Equation (3.22) gives an estimate for $\Omega\left(t_{0}\right)-1$ at present time in terms the density $\rho_{*}$ at inflation, the present density $\rho_{0}$, and the time of inflation $\Delta t$. Again, the formula is independent of the curvature parameter $k$, and hence is independent of the magnitude of the initial curvature $\frac{k}{R_{-}^{2}}$ at the start of inflation $t=t_{-}$. In particular, assuming that $\rho_{*}$ and $\rho_{0}$ are fixed, (3.22) implies that $\left(\Omega\left(t_{0}\right)-1\right)$ will decrease exponentially as $\Delta t$ increases, and the rate is independent of $k$.

\section{Proof:}

To verify (3.22), interchange $t$ and $t_{0}$ in (2.13) to obtain

$$
\begin{aligned}
\Omega\left(t_{0}\right)-1 & =\frac{\Omega(t)-1}{\left\{\Omega(t)\left[\left(\frac{R_{0}}{R}\right)^{2}\left(\frac{\rho_{0}}{\rho}\right)-1\right]+1\right\}} \\
& =\frac{1}{\frac{1}{\Omega(t)-1}\left(\frac{R_{0}}{R}\right)^{2}\left(\frac{\rho_{0}}{\rho}\right)+\left[\left(\frac{R_{0}}{R}\right)^{2}\left(\frac{\rho_{0}}{\rho}\right)-1\right]} .
\end{aligned}
$$

Now if we let subscript zero denote present time, and let subscript + denote the time at the end of inflation, then replacing $t$ by $t_{+}$in (3.23) gives us the relation

$$
\left|\Omega\left(t_{0}\right)-1\right|=\frac{1}{|A \alpha+[\alpha-1]|},
$$


where,

$$
A=\frac{1}{\Omega\left(t_{+}\right)-1}
$$

and

$$
\alpha=\left(\frac{R_{0}}{R_{+}}\right)^{2}\left(\frac{\rho_{0}}{\rho_{*}}\right)
$$

because $\rho_{+}=\rho_{*}$ by (3.1). But using (3.20) we can use (2.10) to estimate

$$
\left(\frac{\rho_{0}}{\rho_{*}}\right)^{\frac{1+3 \sigma}{3(1+\sigma)}} \leq \alpha \leq\left(\frac{\rho_{0}}{\rho_{*}}\right)^{\frac{1}{3}}
$$

which in particular implies that $\alpha<1$. But we always have

$$
\frac{1}{|A \alpha+[\alpha-1]|} \leq \frac{2}{|A| \alpha}
$$

for any $\alpha<1$ and $|A| \alpha>2$, and so assuming this in (3.24) we can estimate

$$
\left|\Omega\left(t_{0}\right)-1\right| \leq \frac{2\left|\Omega\left(t_{+}\right)-1\right|}{\alpha} \leq 16\left(\frac{\rho_{*}}{\rho_{0}}\right)^{\frac{1+3 \sigma}{3(1+\sigma)}} e^{-2 \gamma c \Delta t},
$$

so long as

$$
\left|\Omega\left(t_{+}\right)-1\right|^{-1}>\frac{2}{\alpha}
$$

and

$$
e^{2 \gamma c \Delta t}>4
$$

where we have applied Corollary 1. But by assumption (3.21), which is equivalent to $e^{2 \gamma c \Delta t} \geq \frac{16}{\alpha}>4$, it then follows from (3.17) that

$$
\left|\Omega\left(t_{+}\right)-1\right|^{-1} \geq\left\{\frac{1}{8} e^{2 \gamma c \Delta t}\right\} \geq \frac{2}{\alpha} .
$$

Therefore, (3.29), which is equivalent to (3.22), holds as a consequence of (3.21). This completes the proof of Theorem 4. 
To end this section, we evaluate the constants $\rho_{0}, \rho_{*}$ and $\Delta t$ according to [1]. First consider $\frac{\rho_{*}}{\rho_{0}}$. Since astronomical observations indicate that the universe is flat to within around one percent, we take the critical density at the present value of the Hubble constant as an estimate for $\rho_{0}$. By $(2.5), \rho_{0}$ is then given in terms of the present value of the Hubble constant $H_{0}$ by

$$
\rho_{0} \approx \frac{3 H_{0}^{2}}{\kappa c^{4}}
$$

Here

$$
H_{0}=h_{0} \times \frac{100 k m}{s M p c}
$$

where a megaparsec $M p c$ is given in centimeters by

$$
M p c \approx 3.09 \times 10^{24} \mathrm{~cm},
$$

and $\kappa=\frac{8 \pi \mathcal{G}}{c^{4}}$ where

$$
\frac{\mathcal{G}}{c^{2}} \approx 7.43 \times 10^{-29} \frac{\mathrm{cm}}{\mathrm{g}},
$$

(c.f. [7]). Putting (3.32)-(3.34) into (3.31) gives

$$
\rho_{0} \approx h_{0}^{2} \times 1.9 \times 10^{-29},
$$

where $h_{0}$ is observed to lie between .5 and 1 and is currently taken to be about 0.7 ,

$$
h_{0} \approx 0.7 \text {. }
$$

For the value of $\rho_{*}$ at inflation we take the value given by, ([1], page 541),

$$
\rho_{*} \approx 10^{73} \frac{\mathrm{g}}{\mathrm{cm}^{3}} .
$$

Using (3.35), (3.36) and (3.37) we obtain an estimate for $\frac{\rho_{*}}{\rho_{0}}$ :

$$
\frac{\rho_{0}}{\rho_{*}} \approx 10^{-102}
$$


We can use the values (3.37) and (3.34) in (3.3) to directly estimate $\gamma$ as

$$
\gamma \approx 7.9 \times 10^{22} \mathrm{~cm}^{-1}
$$

A calculation then gives

$$
\gamma c \approx 2.4 \times 10^{33} s^{-1}
$$

Now assuming that $\frac{p(t)}{\rho(t)} \leq \sigma \leq \frac{1}{3}$ for $t \geq t_{+}$, (a reasonable assumption for cosmology, c.f. [7]), so that $\frac{1+3 \sigma}{3(1+\sigma)} \leq \frac{1}{2}$, we obtain the estimate

$$
\left(\frac{\rho_{*}}{\rho_{0}}\right)^{\frac{1+3 \sigma}{3(1+\sigma)}} \leq\left(\frac{\rho_{*}}{\rho_{0}}\right)^{1 / 2} \approx 10^{51} .
$$

Putting (3.40) and (3.41) into (3.22) we obtain

$$
\left|\Omega\left(t_{0}\right)-1\right| \leq 16\left(\frac{\rho_{*}}{\rho_{0}}\right)^{\frac{1}{2}} e^{-2 \gamma c \Delta t} \approx\left(1.6 \times 10^{52}\right) \times e^{-4.8 \times 10^{33} \Delta t},
$$

which holds so long as (3.21) holds, that is

$$
\Delta t \geq \frac{1}{2 \gamma c} \ln 16\left(\frac{\rho_{*}}{\rho_{0}}\right)^{1 / 2} \approx 2.5 \times 10^{-32} .
$$

Finally, by (3.42) it follows that if

$$
\Delta t \leq 2.6 \times 10^{-32}
$$

then

$$
\left|\Omega\left(t_{0}\right)-1\right| \leq 10^{-2}
$$

which by (3.43) is within the range consistent with (3.21). We conclude that if inflation time ends at about $t_{+} \approx 10^{-30} s$ and begins some three orders of magnitude before that time, i.e., at $t_{-}<t_{+}-2.6 \times 10^{-32}$, as in [1], then we will see the present value $\left|\Omega\left(t_{0}\right)-1\right|$ within one percent of unity, confirming the claims in [1].

In conclusion, we have shown that if the inflationary FRW metric (3.2) evolving through the inflationary epoch "connects up" with the the FRW metric of the standard model at the end of inflation, and the time of inflation is at least that given in (3.43), then we have an explanation for why we see 
critical expansion at the present time. Note however that for this to be valid, it requires the assumption that, at the end of inflation, the FRW metric of the standard model really does "connect up" to the inflationary FRW metric at the end of inflation. This requires the assumption that the perfect fluid that emerges from the inflationary spacetime at the end of inflation actually becomes co-moving with respect to the spacelike time slices of the inflationary FRW spacetime at the end of the inflationary epoch. Assuming this, we have shown that, at the end of inflation, the metric will evolve essentially like a $k=0$ FRW metric from that time onward, agreeing with the standard model of cosmology.

\section{References}

[1] S.K. Blau and A.H. Guth, Inflationary cosmology. In: Three Hundred Years of Gravitation, ed. by S.W. Hawking and W. Israel, Cambridge University Press, pp. 524-603 (1987).

[2] S.K. Blau, E.I. Guendelman and A.H. Guth, Dynamics of false vacuum bubbles, Phys. Rev. D, 35, pp. 1747-1766 (1987).

[3] R.H. Dicke and P.J.E. Peebles in General Relativity: An Einstein Centenary Survey, ed. S.W. Hawking and W. Israel, Cambridge University Press, Cambridge, 1979.

[4] P.J.E. Peebles, Principles of Physical Cosmology, Princeton University Press, 1993.

[5] J. Smoller and B. Temple, Shock-wave cosmology inside a black hole, Proceedings of the National Academy of Sciences, 100, pp. 11216-11218 (2003).

[6] R.M. Wald, General Relativity, University of Chicago Press, 1984.

[7] S. Weinberg, Gravitation and Cosmology: Principles and Applications of the General Theory of Relativity, John Wiley \& Sons, New York, 1972. 